\title{
Microsoft erhält für Updateverfahren Datenschutz-Gütesiegel
}

\author{
„Privacy Inside“ aus Kiel für Redmond
}

Johann Bizer

Am 16. Februar 2007 hat das Unabhängige Landeszentrum für Datenschutz SchleswigHolstein an die Microsoft Corporation aus Redmond, USA, ein Datenschutz-Gütesiegel für das Updateverfahren von Microsoft Windows verliehen. Die Verleihung des Gütesiegels wurde durch Ministerpräsident Carstensen (CDU) in der Landesvertretung Schleswig-Holstein in Berlin vorgenommen. Entgegengenommen wurde das Gütesiegel neben Vertretern von Microsoft Deutschland von Peter Cullen, dem Konzernbeauftragten für den Datenschutz für Microsoft weltweit.

Das Gütesiegel ist für die Produkte Microsoft Update Service 6.0 und Windows Server Update Service 2.0 verliehen worden. Diese Systeme dienen dazu, Computer, die mit dem Betriebsystem Windows XP oder Windows Server ausgestattet sind, einfach und effizient mit aktuellen Updates zu versorgen und sie so sicherer zu machen. Aus Sicht des Datenschutzes muss dabei nicht nur verhindert werden, dass unautorisierte Software eingespielt wird, sondern auch, dass das System personenbezogene oder firmeninterne Daten an Microsoft übermittelt, ohne dass der Nutzer derartige Prozesse steuern kann. Die Begutachtung durch die beim ULD anerkannten Prüfstellen TÜV Informationstechnik $\mathrm{GmbH}$ (Technik) sowie dem Unternehmen 2B Secure (Recht) hat ergeben, dass diese Voraussetzungen erfüllt sind.

\section{Premiere}

Diese Verleihung ist eine Premiere. Zum ersten Mal hat das ULD ein DatenschutzGütesiegel international und zugleich für ein Produkt an den größten Hersteller von Software weltweit verleihen können.

Von Bedeutung ist zum einen, dass mit diesem Verfahren europäische Datenschutz- standards von Microsoft explizit als Maßstab für die eigene Produktgestaltung zu Grunde gelegt worden sind. Darüber hinaus ist bemerkenswert, dass sich Microsoft damit auch die führende Rolle von Schleswig-Holstein für den Bereich von Datenschutz und Datensicherheit anerkannt hat.

\section{Ernte einfahren}

Das Datenschutz-Gütesiegel ist ein erfolgreiches Instrument aus Schleswig-Holstein, das seit 2003 vom ULD für IT-Produkte verliehen wird, die in besonderem Maße die Anforderungen des Datenschutzes und der Datensicherheit erfüllen. Es ist in den vier Jahren seines Bestehens bereits 36 Mal für datenschutzfreundliche Produkte verliehen worden. Das Datenschutz-Gütesiegel ist bislang europaweit einzigartig. Die Bemühungen um einen Datenschutz durch Wettbewerb sind im Jahr 2004 das erste Mal international gewürdigt worden, indem das Land Schleswig-Holstein für dieses Konzept von der EU-Kommission einen Innovationspreis erhalten hat.

Mit der Verleihung des Gütesiegels an Microsoft fährt das Land SchleswigHolstein die Ernte für seine Bemühungen um einen modernen und innovativen Datenschutz ein. Die Grundlage hat der Landtag Schleswig-Holstein im Jahr 2001 mit einem im Übrigen einstimmig beschlossenen Landesdatenschutzgesetz gelegt, indem er die Grundlagen für ein technisch-orientiertes Datenschutzzentrum als Dienstleistungszentrum gelegt hat. Im Unterschied zu anderen Ländern ist Schleswig-Holstein nicht auf dem halben Weg eines Ankündigungsgesetzes einer Datenschutz-Zertifizierung stehen geblieben. Insbesondere der Bundesgesetzgeber hat sich seit Jahren trotz mehrerer Aufforderungen durch den Deutschen Bundestag durch Zurückhaltung und
Abstinenz in Sachen Datenschutzzertifizierung ausgezeichnet. §9 a BDSG legt zwar die Grundlagen für ein Datenschutz-Gütesiegel, aber das in dieser Regelung angekündigte Ausführungsgesetz wurde vom Bundesinnenministerium bislang nicht vorgelegt. Die Konzeptlosigkeit des Bundes zeigt, dass der moderne Datenschutz beim Bundesinnenminister offensichtlich in schlechten Händen ist.

\section{Anreize setzen}

Das Gütesiegelverfahren Microsoft macht deutlich, dass das Gütesiegel nicht nur vorbildliche Produkte von kleinen und mittleren Unternehmen auszeichnet, sondern auch als Anreiz von großen Weltkonzernen gesehen wird, seine Produkte an europäischen Datenschutzstandards $\mathrm{zu}$ orientieren. Das Instrument des Datenschutz-Gütesiegels ist eine gelungene Kooperation zwischen Wirtschaft, Verwaltung und Datenschutz, die dem Datenschutz der Bürgerinnen und Bürger zugute kommen soll.

Von der Zertifizierung profitieren alle:

die Hersteller der Produkte, die das Gütesiegel als Wettbewerbsvorteil oder gar Alleinstellungsmerkmal nutzen können,

die Wirtschaft und Verwaltung, der Produkte angeboten werden, bei denen der Datenschutz ,eingebaut“ ist (,Datenschutz inside"),

und allen voran die Bürgerinnen und Bürger, Verbraucherinnen und Verbraucher, die sich sicher sein können, dass mit ihren Daten verantwortungsvoll umgegangen wird.

\section{Dr. Johann Bizer}

Stellvertretender Landesbeauftragter für den Datenschutz Schleswig-Holstein mail@datenschutzzentrum.de 